\title{
Yield of systematic household contact investigation for tuberculosis in a high- burden metropolitan district of South Africa
}

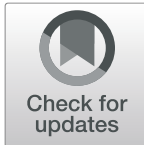

\author{
N. Gladys Kigozi* ${ }^{*}$, J. Christo Heunis and Michelle C. Engelbrecht
}

\begin{abstract}
Background: Systematic household contact investigation ( $\mathrm{SHCl}$ ) is recommended as an active-case-finding (ACF) strategy to identify individuals at high risk of tuberculosis (TB) infection, in order to enable early detection and treatment. Reluctance to implement SHCl in sub-Saharan African and South African high-burden contexts may stem from uncertainty about the potential yield of this strategy when targeting specific categories of TB index cases. In order to inform and motivate scale-up, this pilot study investigated the effectiveness of $\mathrm{SHCl}$ when targeting the World Health Organization's (WHO) recommended categories of infectious index cases.

Method: Data were gathered in September and October 2016. Household contacts of infectious TB cases who attended 40 primary health care facilities in Mangaung Metropolitan District were recruited. The categories of TB index cases included 1) children $<5$ years, 2) HIV co-infected pulmonary TB (PTB) cases ( $\geq 5$ years), 3) HIV-negative PTB cases ( $\geq 5$ years), and 4) multidrug-resistant (MDR) TB cases. Contacts were screened for TB symptoms and symptomatic individuals and all children $<5$ years were referred for clinical evaluation. Data were analysed to establish the yield and factors associated with new TB diagnosis.
\end{abstract}

Results: Of 259 contacts screened, just under half (47.1\%) underwent TB clinical investigation, during which 17 (6.6\%) new TB cases were diagnosed, which represents a prevalence rate of 6564 per 100,000 population. Fifteen contacts needed to be screened to detect one new TB case. The proportion of new TB cases was the highest among contacts of HIV-negative PTB index cases (47.9\%). The likelihood of TB diagnosis was higher among male contacts (odds ratio [OR]: 4.8; 95\% confidence interval [CI]: 1.54-14.97) and those reporting coughing (OR: 4.3; 95\% Cl: $1.11-16.43)$.

Conclusion: The high yield of new TB observed in this pilot study demonstrates that targeted $\mathrm{SHCl}$ may be an effective ACF strategy in Mangaung and similar high-burden settings in South Africa. Targeting different index case categories produced variable yield - the highest among contacts of HIV-negative TB index cases. SHCl among household contacts of all four the WHO-recommended categories of infectious TB index cases - and male and coughing contacts, in particular - should be maximised.

Keywords: Active case finding, Free State Province, Index cases, Household contact investigation, Mangaung Metropolitan District, Tuberculosis

\footnotetext{
* Correspondence: kigozign@ufs.ac.za

Centre for Health Systems Research \& Development, University of the Free State, P.O. Box 399, Bloemfontein 9300, South Africa
}

(c) The Author(s). 2019 Open Access This article is distributed under the terms of the Creative Commons Attribution 4.0 International License (http://creativecommons.org/licenses/by/4.0/), which permits unrestricted use, distribution, and reproduction in any medium, provided you give appropriate credit to the original author(s) and the source, provide a link to the Creative Commons license, and indicate if changes were made. The Creative Commons Public Domain Dedication waiver (http://creativecommons.org/publicdomain/zero/1.0/) applies to the data made available in this article, unless otherwise stated. 


\section{Background}

In 2016, South Africa recorded the highest TB incidence and mortality rates among the 30 high-burden countries, at respectively 781 and 222 per 100,000 population [1]. This pilot study was conducted in the Free State Province, where, in 2016, the TB mortality rate, at 69.7 per 100,000 population, was substantially higher than the national rate of 52.8 per 100,000 population [2]. More specifically, the research took place in Mangaung Metropolitan District, which, in 2016, had a population of 787, 803 [3]. In 2015, the TB incidence rate in Mangaung was higher than the national rate, at 616 and 520 per 100 , 000 population respectively. In 2014, the estimated TBrelated death rate in the district, at $9.7 \%$, was substantially higher than the national rate of $6.1 \%$ [4]. The high TB burden and poor treatment outcomes informed the choice of this province and district for the study.

The World Health Organization (WHO) recommendations for investigating contacts of persons with infectious TB in low- and middle-income countries endorse household contact investigation as one of the activecase-finding (ACF) strategies that can be used to identify individuals at high risk of TB infection in communities [5]. Various recent studies emphasise the importance of household contact investigation [6-9], which enables early TB detection, including identification of latent TB infection, thereby enabling preventive measures and prompt treatment initiation $[5,10]$.

Although policy makers and researchers worldwide recognise the necessity of more aggressive ACF approaches to supplement passive case finding [10-16], robust evidence for the effectiveness of such approaches in highburden sub-Saharan African settings is wanting [17, 18]. Indeed, while the WHO classifies household contact investigation as a "strong recommendation", it concedes that the recommendation is based on "very low-quality evidence" [5], p., 8. At the country level, this may discourage programme-wide implementation of interventions [19].

Systematic screening for active TB involves the systematic identification of people with suspected active TB in a predetermined target group, by using tests, examinations or other procedures that can be applied rapidly [10]. The WHO [5] recommends that contact investigation should be conducted for household and close contacts when the index case has any of the following characteristics: 1) is a child $<5$ years, 2) has HIV co-infected pulmonary TB (PTB) ( $\geq 5$ years), 3 ) has HIV-negative PTB ( $\geq 5$ years) or 4 ) has multidrug-resistant (MDR) TB.

Although TB programmes worldwide have adopted $\mathrm{ACF}$, infrequent or inconsistent investigation of TB patient contacts remains a serious challenge [5, 20-22]. This is also experienced nationally in South Africa [18, 23, 24], and in the Free State province, in particular [25]. Reluctance to implement systematic household contact investigation (SHCI) in the African and South African high-burden contexts may stem from uncertainty about the potential yield of this strategy when targeting specific categories of TB index cases. This study investigated the effectiveness and yield of SHCI when targeting the WHOrecommended categories of infectious TB index cases, in order to inform and motivate SHCI scale-up efforts.

\section{Methods \\ Design and setting}

This pilot study was conducted among purposively-selected household contacts of infectious TB patients attending services at 40 primary health care (PHC) facilities in the Mangaung Metropolitan District.

\section{Participants and sampling}

The study targeted household contacts of infectious TB patients with bacteriologically confirmed TB, or who had started TB treatment based on clinical presentation, $\mathrm{X}$-ray findings or other tests in line with national TB management guidelines. At each $\mathrm{PHC}$ facility, the TB focal person assisted the research team to identify infectious TB index patients. Patients of any age were eligible for recruitment if they were within three months of initiating treatment, reported at least one household contact, had consented to a household visit by the fieldwork team, and had provided a traceable home address. The caretakers of children $<18$ years were approached to provide consent. Index cases who were exclusively on extrapulmonary TB treatment, those who had been on treatment for longer than three months, those who indicated having no household contacts, and those who did not consent to a home visit, were excluded from the study. The WHO-recommended infectious index case categories were purposefully selected at each PHC facility. Efforts were made to recruit at least one index case from each index case category.

Selection of household contacts was also purposive. Household contacts of any age were included in the study if they were not receiving TB treatment at the time of the home visit, had spent at least three months under the same roof as the infectious TB index case, had consented to the study, and were available for interviews during the household visits.

\section{Household visits and data gathering}

Data were gathered between 1 September and 31 October 2016. Figure 1 depicts the process followed during data gathering. Consent procedures were administered to the index cases before they were asked to provide contact details of all members of their household. Fieldworkers sought permission to visit the index cases' households to screen their contacts for TB symptoms, 


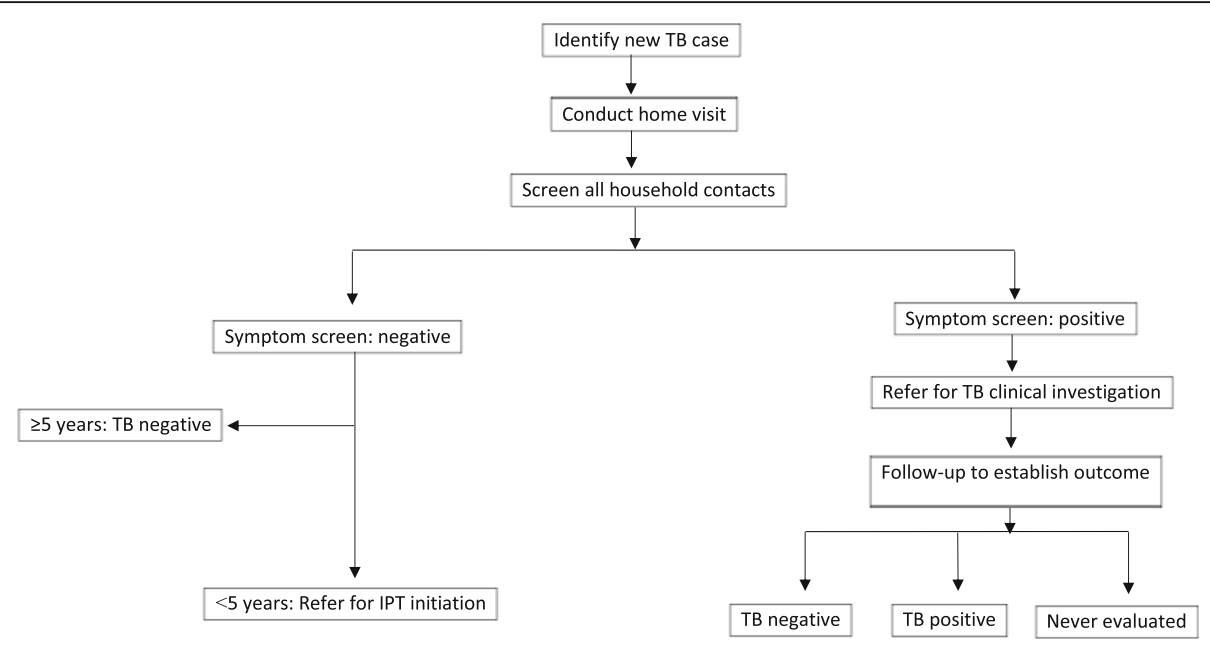

Fig. 1 Procedure. IPT = Isoniazid preventive therapy

and also to extract clinical information from the index cases' medical records at the PHC facilities.

Household visits were conducted within one week of index case recruitment and all contacts available at the time of the visit were considered for the study. Consent was obtained from the household contacts, after which they were screened for TB symptoms, including coughing for longer than two weeks, weight loss, fever and night sweats. In addition to caretaker consent, assent was obtained for children 6-17 years. Demographic and other clinical information, such as uptake of HIV testing, was also collected. Caretakers were interviewed on behalf of children $<18$ years.

In line with TB programme guidelines, all children $<5$ years (regardless of their initial screening results), as well as symptomatic individuals $\geq 5$ years, were referred to the PHC facility for further clinical evaluation. Telephonic follow-up was conducted seven days after the household visits, to determine whether household contacts had actually attended the PHC facility for clinical evaluation and to record the outcome of the evaluation. Where there was non-compliance, fieldworkers made up to two repeat visits to households to encourage the referred contacts to take up clinical investigation.

\section{Statistical analysis}

The data were analysed using SPSS Version 24 and described using frequencies and percentages. The primary outcome was the yield of SHCI, defined as the number of new TB cases identified per household contact screened. The number needed to screen (NNS) in order to identify one new TB case was expressed as the total number of household contacts screened divided by the number of new TB cases. Binary logistic regression was used to examine the association between certain index case and household contact characteristics and new TB diagnosis.
Statistical significance was considered at $p<0.05$ and 95\% confidence interval.

\section{Results}

Figure 2 presents the number of infectious TB index cases and household contacts recruited for the study. A total of 131 index cases were approached, of whom 92 (70.2\%) provided traceable home addresses and were included into the study. The $92(70.2 \%)$ index cases were linked to a total of 297 household contacts. Out of the 297 household contacts, $259(87.2 \%)$ were available for interviews and were also screened for TB symptoms on the day of the field visit. These individuals were included in the analysis. Of the 38 individuals who were not available for interviews, 10 (26.3\%) refused to be interviewed and 28 (73.7\%) had conflicting work or travel commitments.

Table 1 depicts the characteristics of the TB index cases $(n=92)$ and the household contacts screened for TB $(n=259)$. Regarding the index cases, just more than two thirds $(n=63 ; 67.0 \%)$ were male and one quarter $(n=23 ; 25.0 \%)$ were adults aged $35-44$ years. Clinical records showed that just more than one quarter $(n=24$; 26.1\%) of these patients had a history of TB diagnosis and almost all $(n=90 ; 97.8 \%)$ had known HIV status, of whom half $(n=46 ; 50.0 \%)$ were HIV-negative. Further analysis showed that index cases were considerably older than the household contacts: index case median age: 35 years; interquartile range [IQR]: $24-45$ years) vs. contact median age: 20 years (IQR: $8-41$ years). Most $(n=154$; 59.5\%) household contacts were female. The median number of household contacts for every index case was four (IQR: 3-5).

In respect of household contacts, about four in every ten were male $(n=105 ; 40.5 \%)$, and the largest proportion $(n=73 ; 28.2 \%)$ were aged $5-15$ years. About one tenth $(n=33 ; 12.7 \%)$ of the contacts had a history of TB 


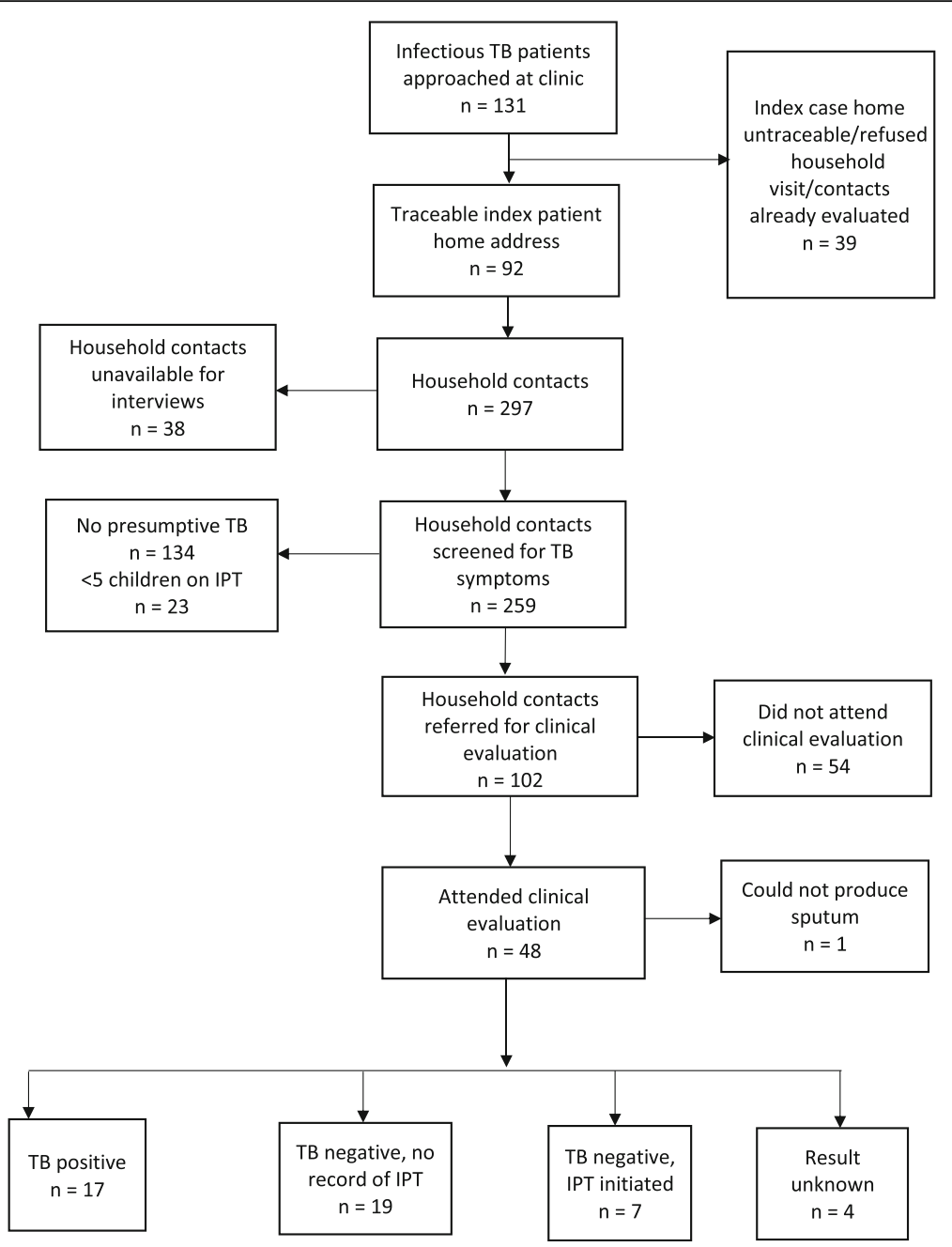

Fig. 2 Flow chart of index cases and household contacts included in the study. IPT = Isoniazid preventive therapy

diagnosis and just over one third $(n=94 ; 36.3 \%)$ shared a bedroom with an infectious index case. A large majority $(n=228 ; 88.0 \%)$ of the contacts self-reported having tested for HIV. However, due to the confidential nature of results, most contacts refused to disclose their HIV status and, for those who did, the results could not be verified and are, therefore, not reported. While two in every five ( $n=102$; $39.4 \%)$ household contacts were referred for clinical evaluation after symptom screening, more than half $(n=54 ; 52.9 \%)$ did not take up this evaluation (Table 1).

Table 2 indicates the yield of SHCI. From the systematic household symptom screening, referral and clinical evaluation exercise, 17 new TB cases were identified. This represents an overall yield of $6.6 \%$ and prevalence rate of 6564 per 100,000 population. The yield ranged from nil among contacts of MDR TB patients (no XDRTB index cases were identified) to $8.0 \%$ among contacts of HIV-negative ( $\geq 5$ years) index cases. For all contacts, the NNS to diagnose one new TB case was 15 . The NNS ranged from 13 among contacts of HIV-negative index cases, to 18 among contacts of HIV-positive patients.

The majority of the 17 new TB cases $(n=12 ; 70.6 \%)$ were male. Male gender was independently statistically significantly (OR: 3.9; CI: 1.31-11.37) associated with the likelihood of a laboratory-confirmed TB diagnosis. After controlling for other variables in the model, male contacts were 4.8 (CI: 1.54-14.97) times more likely to be diagnosed with TB than their female counterparts. Household contacts who self-reported coughing were 4.3 (CI: 1.11-16.43) times more likely to have been diagnosed with TB than those without a cough (Table 3).

\section{Discussion}

This pilot study established a yield of $6.6 \%$ new TB among household contacts where the TB index case 1) is a child < 5 years, 2) has HIV co-infected PTB ( $\geq 5$ years), 3 ) has HIVnegative PTB ( $\geq 5$ years) or 4 ) has MDR TB. A number of 
Table 1 Demographic and clinical characteristics of patients and their household contacts

\begin{tabular}{|c|c|c|c|c|}
\hline \multirow[t]{2}{*}{ Characteristic } & $\begin{array}{l}\text { Index cases } \\
(n=92)\end{array}$ & $\begin{array}{l}\text { Contacts TB screened } \\
(n=259)\end{array}$ & $\begin{array}{l}\text { Contacts referred for clinical } \\
\text { evaluation }(n=102)\end{array}$ & $\begin{array}{l}\text { Contacts who underwent clinical } \\
\text { evaluation }(n=48)\end{array}$ \\
\hline & n (\%) & n (\%) & n (\%) & n (\%) \\
\hline Male & $63(68.5)$ & $105(40.5)$ & $48(47.1)$ & $26(54.2)$ \\
\hline \multicolumn{5}{|l|}{ Age in years ${ }^{a}$} \\
\hline$<5$ & $12(13.0)$ & $36(13.9)$ & $13(12.7)$ & $8(16.7)$ \\
\hline $5-15$ & $3(3.3)$ & $73(28.2)$ & $28(27.5)$ & 15 (31.3) \\
\hline $16-24$ & $8(8.7)$ & $33(12.7)$ & $15(14.7)$ & $6(12.5)$ \\
\hline $25-34$ & $22(23.9)$ & $34(13.1)$ & $14(13.7)$ & $5(10.4)$ \\
\hline $35-44$ & $23(25.0)$ & $25(9.7)$ & $7(6.9)$ & $2(4.2)$ \\
\hline $45-54$ & $13(14.1)$ & $19(7.3)$ & $8(7.8)$ & $4(8.3)$ \\
\hline $55-64$ & $7(7.6)$ & $18(7.0)$ & $6(5.9)$ & $3(6.3)$ \\
\hline $65+$ & $4(4.3)$ & $21(8.1)$ & $11(10.8)$ & $5(10.4)$ \\
\hline $\begin{array}{l}\text { Previously diagnosed with } \\
\text { TB }\end{array}$ & $22(24.2)$ & $33(12.7)$ & 21 (19.6) & $8(16.7)$ \\
\hline $\begin{array}{l}\text { Shares bedroom with TB } \\
\text { index case }\end{array}$ & & $94(36.3)$ & $52(44.1)$ & 19 (39.6) \\
\hline $\begin{array}{l}\text { Ever tested for HIV (self- } \\
\text { report) }\end{array}$ & $92(100)$ & $228(88.0)$ & $101(82.4)$ & 43 (89.6) \\
\hline \multicolumn{5}{|l|}{ HIV test result ${ }^{\mathrm{b}}$} \\
\hline HIV-positive & $44(47.8)$ & & & \\
\hline HIV-negative & $46(50.0)$ & & & \\
\hline Not recorded & $2(2.2)$ & & & \\
\hline
\end{tabular}

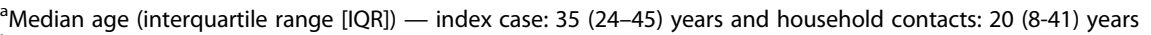

${ }^{\text {b}}$ TB index case HIV status was verified from medical records at PHC facility; contacts' HIV status was self-reported and could not be verified

previous studies in sub-Saharan African countries examined the yield of TB among household contacts of different categories of TB index cases. Two sub-Saharan African country studies established a yield of $10 \%$ or higher new TB among household contacts - a Ugandan study targeting new sputum smear positive $\left(\mathrm{SS}_{+}\right)$index TB cases $(\geq 15$ years): $15.7 \%$ [26]; and an Ethiopian study of MDR TB index cases (all ages): 10.0\% [27]. Other sub-Saharan African country studies produced a yield of between 5 and $8 \%$ - a study in North West Province of South Africa targeting newly diagnosed TB index cases ( $\geq 15$ years): $7.8 \%$ [28]; a Kenyan study targeting SS+ TB cases (all ages): 6.7\% [29]; an Ethiopian study targeting all types of TB cases $(\geq 18$ years): $6.5 \%$ [30]; and a Tanzanian study targeting laboratory-confirmed TB cases (all ages): 6.4\% [12].

The $6.6 \%$ yield of new TB among the household contacts of the WHO-recommended infectious index case categories for SHCI observed in the current study, falls within the typical range of 5 to $8 \%$ observed in subSaharan African country studies utilising a targeted SHCI approach. Thereupon non-targeted household contact investigation studies in sub-Saharan African countries tend to demonstrate a lower yield. For example, a study in the North West Province of South

Table 2 Yield of systematic household contact investigation

\begin{tabular}{|c|c|c|c|c|}
\hline Variable & Household contacts screened $n$ & Number of new TB cases $n$ & Yield \% & NNS \\
\hline All participants & 259 & 17 & 6.6 & 15 \\
\hline \multicolumn{5}{|l|}{ Index case category } \\
\hline Children $<5$ years & 29 & 2 & 6.9 & 14 \\
\hline HIV-positive ( $\geq 5$ years) & 124 & 7 & 5.6 & 18 \\
\hline HIV-negative ( $\geq 5$ years) & 100 & 8 & 8.0 & 13 \\
\hline MDR-TB ( $\geq 5$ years) & 6 & 0 & 0 & a \\
\hline
\end{tabular}

anly one contact was symptomatic, clinical evaluation did not yield new TB 
Table 3 Factors associated with likelihood for new TB diagnosis among household contacts

\begin{tabular}{|c|c|c|c|}
\hline Variable & $\begin{array}{l}\text { Diagnosed with TB } \\
(n=17) \\
\mathrm{n}(\%)\end{array}$ & Unadjusted OR (95\% Cl) & Adjusted OR (95\% Cl) \\
\hline \multicolumn{4}{|l|}{ Sex } \\
\hline Female (ref) & $5(29.4)$ & 1 & 1 \\
\hline Male & $12(70.6)$ & $3.9(1.31-11.37)$ & $4.8(1.54-14.97)$ \\
\hline Age (median; IQR) & $18(7-43)$ & $1.0(0.97-1.02)$ & $1.0(0.97-1.01)$ \\
\hline Number of household contacts from same household (median; IQR) & $4(2-5)$ & $1.0(0.76-1.31)$ & $1.0(0.76-1.32)$ \\
\hline \multicolumn{4}{|l|}{ Whether index case is coughing } \\
\hline No (ref) & $5(29.4)$ & 1 & 1 \\
\hline Yes & $12(70.6)$ & $2.2(0.74-6.36)$ & $2.8(0.84-9.05)$ \\
\hline \multicolumn{4}{|l|}{ Index case HIV status } \\
\hline Negative (ref) & $10(58.8)$ & 1 & 1 \\
\hline Positive & $7(41.2)$ & $0.5(0.18-1.32)$ & $0.6(0.19-1.60)$ \\
\hline \multicolumn{4}{|l|}{ Whether contact shares bedroom with index case } \\
\hline No (ref) & $8(47.1)$ & 1 & 1 \\
\hline Yes & $9(52.9)$ & $2.2(0.81-6.10)$ & $2.3(0.78-6.55)$ \\
\hline \multicolumn{4}{|l|}{ Whether household contact is coughing } \\
\hline No (ref) & $13(76.5)$ & 1 & 1 \\
\hline Yes & $4(23.5)$ & $2.9(0.88-9.73)$ & $4.3(1.11-16.43)$ \\
\hline
\end{tabular}

Ref reference, IQR interquartile range, $\mathrm{Cl}$ confidence interval

Africa among adult TB index cases produced a yield of only $1.3 \%$ [31] and a study in Ghana among adult TB index cases returned a yield of only $0.65 \%$ [32].

The current study did not yield any new TB among household contacts of MDR TB index cases. A systematic review and meta-analysis [7] of the yield of active TB among household contacts of mono, poly, multi or extensively drug-resistant TB index cases recorded in 25 studies in 13 countries, including three studies in South Africa, showed that the yield varied from nil recorded in studies among adults in Taiwan [33] and Switzerland [34], to $23 \%$ recorded in a South African study of children evaluated as household contacts of adult MDR TB index cases in the Western Cape Province [35]. The lack of yield among contacts of MDR TB index cases in the current study could possibly be attributed to the fact that only one MDR TB patient contact was screened to be symptomatic and referred for clinical evaluation, subsequently yielding no TB.

Similar to findings of a study in India [36], and another in the North West Province of South Africa [28], the yield of new TB cases in the current study was highest among contacts of HIV-negative index cases. This finding could potentially be linked to the programmatic drive and recommendation for routine $\mathrm{TB}$ screening and evaluation of HIV-positive individuals, as they are particularly vulnerable and prone to the negative effects of late diagnosis and treatment of TB [6]; consequently, less attention may be given to HIV-negative TB patients. The finding of high prevalence of TB among household contacts of HIV-negative index cases underscores the need for TB programmes to direct ACF efforts, i.e., SHCI, beyond children $<5$ years and HIV-positive cases, and to consider a broader range of risk categories.

Practical experience has shown that, in order to prevent laboratory overload, an average of seven (range: 3-20) people with presumptive TB should be screened to identify one SS+ case of TB [37]. In a resource-constrained country, such as South Africa, the NNS is particularly useful to determine the effectiveness of household contact screening within the context of resource scarcity [37, 38]. The NNS to diagnose one new TB case in the current study was 15 , which lies within the range of 4-71 reported in a systematic review of contact tracing studies across Africa, Asia and the Americas [37]. The finding highlights the need to prioritise targeted SHCI as a strategy for ACF.

While there is widespread evidence that SHCI is effective, it is important that symptomatic and high-risk contacts present promptly at health facilities for clinical evaluation $[5,21]$. In the current study, despite consistent efforts by the research team to follow up and encourage household contacts to attend clinical evaluation following non-compliance, less than half (47\%) of those referred, underwent clinical evaluation. As reported by an earlier study in the Mangaung Metropolitan District [25], this failure to present was primarily due to individual (as opposed to health systems) reasons, such as lack of time, perceived inability to produce a sputum sample, 
lack of transport to clinics, and work or travel commitments. Similarly, in a Ugandan study, individual factors, including fear of stigma, limited knowledge, mistrust of health workers by both TB index cases and their contacts, and lack of time to travel to health facilities, emerged as key barriers to non-completion of clinical evaluation by contacts [21].

Ultimately, $\mathrm{SHCI}$ is about increasing early access to $\mathrm{TB}$ services. This pilot study suggests that alternative strategies by the TB programme in the Mangaung Metropolitan District, to ensure that symptomatic and high-risk contacts complete clinical evaluation, as well as efforts to ensure that they are linked to effective treatment and sustained care, may be necessary. In this study, efforts were made by fieldworkers to follow up and encourage those who had not undergone clinical evaluation, to do so. Under routine programme conditions, this necessitates dedicated human resources to conduct routine household screening coupled with continuous follow-up, to ensure clinical evaluation and treatment initiation. Previous research in South Africa [39] and Kenya [40] found that counselling by community health workers (CHWs) can have a positive impact on uptake of HIV counselling and testing services. A previous study among TB patients in the Free State established their satisfaction with and even preference for HIV counselling and testing delivery by CHWs instead of nurses [41].

The following limitations are associated with the current study. First, this was a pilot study, conducted over of a period of only 2 months, to gauge the effectiveness and yield of systematic screening and referral of household contacts for TB evaluation, in order to inform scale-up efforts in the Free State Province. Under ideal conditions, contacts can be visited and motivated more than twice, until they decide to undertake clinical evaluation. Second, due to purposive sampling of infectious index cases and their household contacts, the results are not generalisable to all contacts of TB patients in the province. Third, to allow for prompt referral, fieldwork was conducted within PHC facility operating hours. Some household contacts were missed, as the fieldwork teams were not able to conduct household visits during the evenings and over weekends. Fourth, due to lack of expertise and resources, the research team could not collect sputum samples from symptomatic contacts, and these contacts, therefore, had to be referred to PHC facilities. Similarly, asymptomatic contacts were not tested for HIV to determine their eligibility for Isoniazid preventive therapy (IPT). Thus, only children $<5$ years were referred for clinical evaluation and/or IPT initiation at the PHC facilities.

\section{Conclusion}

This pilot study shows that targeted SHCI using the WHO-recommended infectious TB index case categories can be effective. The results confirm the need to direct ACF efforts, such as SHCI beyond children $<5$ years and HIV-positive cases, and to consider a broader range of risk categories. Results showed variation in the yield of $\mathrm{TB}$ across the different WHO-recommended TB index case categories, with the highest yield among contacts of HIVnegative PTB index cases, which are not currently prioritised. Routine SHCI of household contacts might improve the yield of ACF among HIV-negative TB and other highrisk infectious index cases and, particularly, as observed in this pilot study, male and coughing household contacts. For Mangaung and similar high-risk settings, this necessitates dedicated human resources towards routine $\mathrm{SHCI}$, and concerted efforts to follow up contacts for clinical evaluation for TB.

\section{Abbreviations}

ACF: Active case finding; CHW: Community health worker; HIV: Human immunodeficiency virus; IPT: Isoniazid preventive therapy; MDRTB: Multidrug-resistant tuberculosis; NNS: Number needed to screen; PHC: Primary health care; PTB: Pulmonary tuberculosis; SHCl: Systematic household contact investigation; SS+: Sputum smear-positive;

TB: Tuberculosis; WHO: World Health Organization

\section{Acknowledgements}

The facilitating role of the Free State Department of Health and the participation of the individual PHC facilities, nurses, TB index cases and household contacts - as well as the efforts of the fieldwork team - are gratefully acknowledged.

\section{Authors' contributions}

NGK drafted the initial manuscript, analysed data, and revised subsequent manuscripts. JCH and MCE contributed to the study design. JCH and NGK revised the manuscript in response to the reviews. All authors read and approved the manuscript.

\section{Funding}

This research is partially funded by an African Doctoral Dissertation Research Fellowship Re-entry grant awarded by the African Population and Health Research Centre in partnership with the International Development Research Centre (Grant No: RCS/ADDRF/2014/131), as well as the National Research

Foundation of South Africa (Grant No. 94116). The funders did not contribute to the study design, data collection and writing of the manuscript.

\section{Availability of data and materials}

The data analysed during this study are not publicly available, as individual privacy could be compromised.

\section{Ethics approval and consent to participate}

Ethical clearance was granted by the Ethics Committee of the Faculty of Health Sciences (IRB00006240), University of the Free State. Authorisation to conduct research at PHC facilities was granted by the Free State Department of Health. Participation in the study was voluntary. Participants were informed about the study and written consent was obtained from all participants $\geq 18$ years, and from caretakers on behalf of those aged $<18$ years. Assent was also sought from children aged 6-17 years.

Consent for publication

Not applicable in this section.

Competing interests

The authors declare that they have no competing interests. 
Received: 27 September 2018 Accepted: 18 June 2019 Published online: 03 July 2019

\section{References}

1. World Health Organization (WHO). Global tuberculosis report 2017. Geneva: WHO; 2017.

2. Day C, Gray A. Health and related indicators. In: Rispel LC, Padarath A, editors. South African health review 2018. Durban: Health Systems Trust; 2018. p. 139-250.

3. Statistics South Africa (Stats SA). Provincial profile: Free State, Community Survey 2016. Pretoria: Stats SA; 2018.

4. Massyn N, Peer N, English R, Padarath A, Barron P, Day C. District health barometer 2015/16. Durban: Health Systems Trust; 2016.

5. World Health Organization (WHO). Recommendations for investigating contacts of persons with infectious tuberculosis in low- and middle-income countries. Geneva: WHO; 2012.

6. Fox GJ, Barry SE, Britton WJ, Marks GB. Contact investigation for tuberculosis: a systematic review and meta-analysis. Eur Respir J. 2013;41(1):140-56.

7. Shah NS, Yuen CM, Heo M, Tolman AW, Becerra MC. Yield of contact investigations in households of patients with drug-resistant tuberculosis: systematic review and meta-analysis. Clin Infect Dis. 2014;58(3):381-91.

8. Bartu V. Importance of TB contact investigations. Respir Med Case Rep. 2016;18:87-9.

9. Jerene D, Melese M, Kassie Y, Alem G, Daba SH, Hiruye N, et al. The yield of a tuberculosis household contact investigation in two regions of Ethiopia. Int J Tuberc Lung Dis. 2015;19(8):898-903.

10. World Health Organization (WHO). Systematic screening for active tuberculosis: principles and recommendations. Geneva: WHO; 2013.

11. National Department of Health (NDoH). Tuberculosis Management Guidelines. Pretoria: NDoH; 2014.

12. Beyanga M, Kidenya BR, Gerwing-Adima L, Ochodo E, Mshana SE, Kasang C. Investigation of household contacts of pulmonary tuberculosis patients increases case detection in Mwanza City, Tanzania. BMC Infect Dis. 2018;18:110.

13. World Health Organization (WHO). Guidelines for intensified tuberculosis case-finding and isoniazid preventive therapy for people living with HIV in resource constrained settings. Geneva: WHO; 2011.

14. Azman AS, Golub JE, Dowdy DW. How much is tuberculosis screening worth? Estimating the value of active case finding for tuberculosis in South Africa, China, and India. BMC Med. 2014;12:216.

15. Yaesoubia R, Cohen T. Identifying dynamic tuberculosis case-finding policies for HIV/TB coepidemics. Proc Natl Acad Sci U S A. 2013;110(23):9457-62.

16. Abebe M, Doherty M, Wassie L, Demissie A, Mihret A, Engers H, et al. TB case detection: can we remain passive while the process is active? Pan Afr Med J. 2012:11:50.

17. Fox GJ, Nhung NV, Sy DN, Hoa NLP, Anh LTN, Anh NT, et al. Householdcontact investigation for detection of tuberculosis in Vietnam. N Engl J Med. 2018;378(3):221-9.

18. Little KM, Msandiwa R, Martinson N, Golub J, Chaisson R, Dowdy D. Yield of household contact tracing for tuberculosis in rural South Africa. BMC Infect Dis. 2018;18(1):299.

19. Cobelens F, van Kampen S, Ochodo E, Atun R, Lienhardt C. Research on implementation of interventions in tuberculosis control in low- and middleincome countries: a systematic review. PLoS Med. 2012;9(12):e1001358.

20. Ayakaka I, Ackerman S, Ggita JM, Kajubi P, Dowdy D, Haberer JE, et al. Identifying barriers to and facilitators of tuberculosis contact investigation in Kampala, Uganda: a behavioral approach. Implement Sci. 2017;12:33.

21. Armstrong-Hough M, Turimumahoro P, Meyer AJ, Ochom E, Babirye D, Ayakaka I, et al. Drop-out from the tuberculosis contact investigation cascade in a routine public health setting in urban Uganda: a prospective, multi-center study. PLoS One. 2017;12(11):e0187145.

22. Hwang TJ, Ottmani S, Uplekar M. A rapid assessment of prevailing policies on tuberculosis. Int J Tuberc Lung Dis. 2011;15(12):1620-2.

23. National Department of Health (NDoH)/World Health Organization (WHO). Joint Department of Health and WHO TB programme review. Pretoria: $\mathrm{NDoH} ; 2014$

24. Churchyard GJ, Mametja LD, Mvusi L, Ndjeka N, Hesseling AC, Reid A. Tuberculosis control in South Africa: successes, challenges and recommendations. S Afr Med J. 2014;104((3) Suppl 1):244-8.
25. Kigozi G, Engelbrecht M, Heunis C, Janse van Rensburg A. Household contact non-attendance of clinical evaluation for tuberculosis: a pilot study in a high burden district in South Africa. BMC Infect Dis. 2018;18:106.

26. Bonnet M, Kyakwera C, Kyomugasho N, Atwine D, Mugabe F, Nansumba M, et al. Prospective cohort study of the feasibility and yield of household child tuberculosis contact screening in Uganda. Int J Tuberc Lung Dis. 2017;21(8):862-8.

27. Titiyos A, Jerene D, Enquselasie F. The yield of screening symptomatic contacts of multidrug-resistant tuberculosis cases at a tertiary hospital in Addis Ababa, Ethiopia. BMC Res Notes. 2015;8:501.

28. Shapiro AE, Variava E, Rakgokong MH, Moodley N, Luke B, Salimi S, et al. Community based targeted case finding for tuberculosis and HIV in household contacts of patients with tuberculosis in South Africa. Am J Respir Crit Care Med. 2012;185:1110-6.

29. Volkmann T, Okelloh D, Agaya J, Cain K, Ooko B, Malika T, Burton D. Pilot implementation of a contact tracing intervention for tuberculosis case detection in Kisumu County, Kenya. Public Health Action. 2016;6(4):217-9.

30. Gebregergs GB, Alemu WG. Household contacts screening adherence among tuberculosis patients in northern Ethiopia. PLoS One. 2015;10(5):e0125767.

31. Van Schalkwyk C, Variava E, Shapiro AE, Rakgokong M, Masonoke K, Lebina $L$, et al. Incidence of TB and HIV in prospectively followed household contacts of TB index patients in South Africa. PLoS One. 2014;9(4):e95372.

32. Ohene SA, Bonsu F, Hanson-Nortey NN, Sackey A, Danso S, Afutu F, et al. Yield of tuberculosis among household contacts of tuberculosis patients in Accra, Ghana. Infect Dis Poverty. 2018;7:14.

33. Huang YW, Shen GH, Lee JJ, Yang WT. Latent tuberculosis infection among close contacts of multidrug-resistant tuberculosis patients in Central Taiwan. Int J Tuberc Lung Dis. 2010;14:1430-5.

34. Younossian AB, Rochat T, Ketterer JP, Wacker J, Janssens JP. High hepatotoxicity of pyrazinamide and ethambutol for treatment of latent tuberculosis. Eur Respir J. 2005:26:462-4.

35. Schaaf HS, Gie RP, Kennedy M, Beyers N, Hesseling PB, Donald PR. Evaluation of young children in contact with adult multidrug-resistant pulmonary tuberculosis: a 30-month follow-up. Pediatrics. 2002;109:765771.

36. Nair D, Rajshekhar N, Shyam Klinton J, Watson B, Velayutham B, Tripathy JP, et al. Household contact screening and yield of tuberculosis cases - a clinic based study in Chennai, South India. PLoS One. 2016;11(9):e0162090.

37. Kranzer K, Houben RM, Glynn JR, Bekker LG, Wood R, Lawn SD. Yield of HIVassociated tuberculosis during intensified case finding in resource-limited settings: a systematic review and meta-analysis. Lancet. 2010;10:93-102.

38. Sekandi JN, List J, Luzze H, Yin X-P, Dobbin K, Corso PS, et al. Yield of undetected tuberculosis and human immunodeficiency virus coinfection from active case finding in urban Uganda. Int J Tuberc Lung Dis. 2014;18(1):13-9.

39. Mkwanazi NB, Patel D, Newell M-L, Rollins NC, Coutsoudis A, Coovadia HM, et al. Rapid testing may not improve uptake of HIV testing and same day results in a rural south African community: a cohort study of 12,000 women. PLoS One. 2008;3(10):e3501.

40. Arthur GR, Ngatia G, Rachier C, Mutemi R, Odhiambo J, Gilks CF. The role for government health centers in provision of same-day voluntary HIV counseling and testing in Kenya. J Acquir Immune Defic Syndr. 2005;40:329-35

41. Kigozi NG, Heunis JC, van den Berg HS. Tuberculosis patients' perspectives on HIV counselling by lay counsellors Vis-à-Vis counselling rendered by nurses: an exploratory study in two districts of the Free State Province, South Africa. Afr J Nurs Midwifery. 2011;13:71-80.

\section{Publisher's Note}

Springer Nature remains neutral with regard to jurisdictional claims in published maps and institutional affiliations. 